\title{
Anatomical connections of the periaqueductal gray: specific neural substrates for different kinds of fear
}

D.M.L. Vianna and M.L. Brandão
Laboratório de Psicobiologia, Faculdade de Filosofia, Ciências e Letras de Ribeirão Preto, Universidade de São Paulo, Ribeirão Preto, SP, Brasil

\author{
Correspondence \\ D.M.L. Vianna \\ Laboratório de Psicobiologia \\ FFCLRP, USP \\ Av. Bandeirantes, 3900 \\ 14040-901 Ribeirão Preto, SP \\ Brasil \\ Fax: +55-16-602-3632 \\ E-mail: dmvianna@usp.br \\ Research supported by FAPESP \\ (Nos. 97/00363-1 and 98/11187-2) \\ and CNPq (No. 140164/1999-5). \\ D.M.L. Vianna is the recipient \\ of a fellowship from $\mathrm{CNPq}$.
}

Received August 5, 2002

Accepted February 14, 2003

\begin{abstract}
The periaqueductal gray (PAG) has been traditionally considered to be an exit relay for defensive responses. Functional mapping of its subdivisions has advanced our knowledge of this structure, but synthesis remains difficult mainly because results from lesion and stimulation studies have not correlated perfectly. After using a strategy that combined both techniques and a reevaluation of the available literature on PAG function and connections, we propose here that freezing could be mediated by different PAG subdivisions depending on the presence of immediate danger or exposure to related signaling cues. These subdivisions are separate functional entities with distinct descending and ascending connections that are likely to play a role in different defensive responses. The existence of ascending connections also suggests that the PAG is not simply a final common path for defensive responses. For example, the possibility that indirect ascending connections to the cingulate cortex could play a role in the expression of freezing evoked by activation of the neural substrate of fear in the dorsal PAG has been considered.
\end{abstract}

Key words

- Periaqueductal gray

- Defense reaction

- Fear

- Escape

- Freezing

- Periaqueductal gray columns

\section{Introduction}

Since Hunsperger (1), the periaqueductal gray (PAG) has been viewed as the final common path for all defensive responses. Chemical or electrical stimulation of some structures located more rostrally, like the amygdala or medial hypothalamus, can induce defense reactions (2), which are permanently abolished after lesion of the PAG (1). The reverse is not true, because neither telencephalic ablation (3) nor medial or posterior hypothalamic lesions (1) blocks defense reactions elicited by PAG stimulation. PAG lesions can also abolish or cause dramatic reductions of both innate (4) and learned (5) defensive behaviors. These findings were later supplemented by the discovery of the organization of the PAG into longitudinal columns, which serve as distinct anatomical modules for the specific functions associated with this structure (6). Presently, four main longitudinal cell-rich subdivisions are accepted by consensus to exist in the PAG (7), namely the dorsomedial (dmPAG), dorsolateral (dlPAG), lateral and ventrolateral (vlPAG) subdivisions. In this review, we examine the special role played by these distinct columns in the coordination of different types of fear in an attempt to present 
an overview of the anatomical organization of the PAG involved in the expression of emotional behavior.

\section{Periaqueductal gray subdivisions: functional differentiation}

It was only in the late eighties that functional studies started to draw attention to regional dissimilarities within the PAG. LeDoux et al. (5) observed a reduction of conditioned freezing behavior after excitotoxic lesion of the caudal, but not the rostral PAG. In further studies of the rostrocaudal axis, stimulation of the caudal PAG produced forward escape, while rostral stimulation provoked threatening reactions and backward avoidance (8), as seen in Figure 1A.

On the dorsoventral axis, electrical stimulation of the dorsal and lateral PAG of the rat provoked vigorous motor reactions similar to those induced by foot shock, and shortly after the end of the stimulus the rat froze (9). In contrast, only freezing was observed after vlPAG stimulation (9). Ventral PAG lesions reduced conditioned freezing, and dorsal PAG lesions reduced the "activity burst" that appears during foot shock (9). In short, it was argued that the dorsal PAG mediated escape, while the ventral PAG was responsible for the freezing response (9).
Some additional support for this view (8) came from the fact that lateral PAG stimulation with excitatory amino acids provoked vigorous escape, while vlPAG stimulation produced strong immobility (Figure 1A).

The findings described above also raised some points for discussion. Microinjection of glutamate into the vlPAG causes an immobile behavior (10) that is very different from the defensive freezing attenuated after lesioning the same region (9). While the former is accompanied by hypotension, bradycardia, and hyporeactivity (10), freezing is followed by hypertension (11) and hypervigilance (12).

Recently, Morgan and Carrive (13) argued against the assumption that vIPAG stimulation causes both immobility and hypotension, and also against the suggestion (10) that this immobility was part of a recuperative response after an agonistic encounter (quiescence). After microinjection of $d, l$ homocysteic acid into the vlPAG of awake, freely moving rats, the resulting immobility and bradycardia were not accompanied by a fall in blood pressure. These investigators concluded that, in nature, generation of behavior usually requires the activation of various brain regions and that the vIPAG could be recruited in both defensive freezing and quiescence. This co-activation hypothesis
Figure 1. Functional studies. A, Sites of excitatory amino acid microinjections into the periaqueductal gray and behavioral reactions thus elicited in the freely moving rat. $B$, Periaqueductal gray neurons showing conditioned fear-related Fos expression. III, oculomotor nucleus; EW, Edinger-Westphal nucleus; MLF, medial longitudinal fasciculus. Reprinted respectively from Carrive (8) and Carrive et al. (18) (c) 1993, 1997, with permission of Elsevier Science.

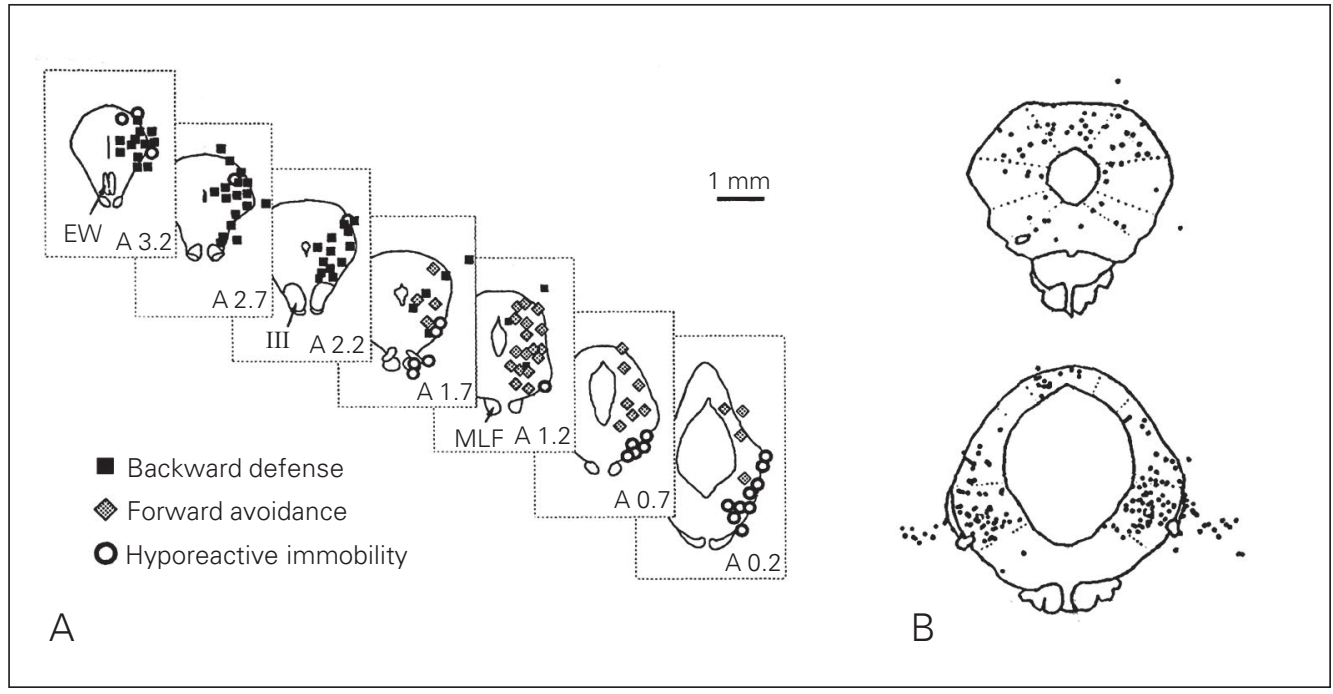


receives support from various correlation studies. Fos immunoreactivity expressed during neuronal activation increases in all PAG subdivisions after stimulation of the dorsal PAG (14) and medial hypothalamus (15), after exposure to a predator (16) or to its smell (17), and after re-exposure to a conditioned aversive context (18). However, it is noteworthy that the effect is much more pronounced in the dIPAG after predator exposure, and in the vlPAG after re-exposure to the conditioned aversive context (Figure 1B).

Yet, there remains a second difficulty with the anatomical dissociation theory. Freezing occurs during electrical $(19,20)$ and chemical $(21,22)$ stimulation of the same PAG sites where escape is elicited, often before it. Freezing induced by stimulation is behaviorally similar to conditioned freezing, as both occur concomitantly with muscular tension, hypervigilance and hyperreactivity (22). Therefore, these findings suggest that the neural substrates for freezing and escape may overlap.

Fanselow (9) suggested that freezing observed after dorsal PAG stimulation is similar to that observed after foot shock. This would imply that dorsal PAG stimulation, which is known to be aversive (23) and to cause conditioning (24), could be responsible for freezing by means of contextual conditioning, i.e., stimulation of PAG sites that give rise to escape would function as an unconditioned aversive stimulus, that would become associated with the contextual cues present during the experimental session. Once associated with the stimulation, these cues would provoke a conditioned fear response in the rat, i.e., freezing behavior. This interpretation is not valid because when we remove the animal from the environment where the experimental session took place, the freezing behavior persists for a significant period of time, and vanishes after $24 \mathrm{~h}$ (25).

Another possible explanation consistent with the hypothesis of dissociated anatomical substrates for freezing and escape would be the stimulus propagation from dorsal PAG to vlPAG. This hypothesis has not received empirical support. After vlPAG stimulation with intensities high enough to provoke escape, animals did not freeze beyond control levels, as occurred with dIPAG stimulation (26).

Finally, the vlPAG does not seem to be critical to all kinds of freezing (27). While vlPAG-lesioned animals did freeze less after foot shock conditioning, electrical or chemical stimulation of the dlPAG in vlPAGlesioned animals caused as much freezing and escape as that shown by sham-lesioned, dlPAG-stimulated animals. Based on this, it has been suggested that the vIPAG is part of a circuit that mediates conditioned fear, while unconditioned fear would use an alternate route that may include the dIPAG.

\section{Connections of periaqueductal gray subdivisions}

The connections between PAG and other structures of the central nervous system are not homogeneous throughout, suggesting some regional specialization. A careful analysis of these connections may indicate the functions performed by each subregion. We describe below the structures that maintain connections with the PAG, selected according to three criteria: a) having strong or moderate monosynaptic connections with the PAG; b) being implicated in the mediation of somatic defense reactions, and c) whose connections are unequally distributed between PAG subregions. It should be pointed out that data obtained from radioligand assays were not considered in the present review because we believe that staining techniques on slices are more precise anatomically.

\section{Connections with higher structures}

\section{Hypothalamus}

The hypothalamus has traditionally been thought to act as a key effector center for 
visceral defense reactions (5), but a long line of inquiry also points at its relevance for the mediation of defensive somatic responses. Amongst the most important lines of evidence is the escape provoked by microinjection of GABA antagonists into the dorsomedial hypothalamus (DMH; 28), an effect that could persist after telencephalic ablation (3). Canteras (29) called attention to a network of hypothalamic subnuclei that may influence the expression of defensive behavior. This network appears to be composed by the anterior hypothalamic nucleus (AHN), the dorsomedial part of the ventromedial hypothalamic nucleus (VMH) and the dorsal premammillary nucleus (PMd). Cell body-spe- cific chemical lesions of the caudal pole of this network (PMd) virtually abolished freezing and escape responses in the presence of a predator (29). All of these areas have increased c-Fos expression during predator odor exposure (17) or after escape provoked by microinjection of nitric oxide donors into the dIPAG (30). Other studies have also suggested the participation of these regions in conditioned fear (31) and in the responses elicited by aversive stimulation of the medial hypothalamus (15) and dorsal PAG (14).

Cameron et al. (32) compared the projections of neurons located in rostral and caudal portions of the dIPAG and vlPAG and observed that the rostral dlPAG densely projects
Figure 2. Orthogradely labeled terminations from afferent structures to the periaqueductal gray. Large dots in $\mathrm{F}$ and $\mathrm{G}$ represent retrogradely labeled neuronal somata. III, oculomotor nucleus; dl, dorsolateral periaqueductal gray; $\mathrm{dm}$, dorsomedial periaqueductal gray; EW, Edinger-Westphal nucleus; I, lateral periaqueductal gray; ND, nucleus of Darkschewitsch; PAG, periaqueductal gray; RL, rostral linear raphe nucleus; vl, ventrolateral periaqueductal gray. $A$, Reprinted from Canteras and Swanson (34), with permission. $B, C, F$ and $G$, Reprinted from Newman et al. (42) with permission of S. Karger AG. $D$ and $H$, Reprinted from Floyd et al. (43), and I, from Vertes (46), () 2000, 1991, with permission of Wiley-Liss, Inc., a subsidiary of John Wiley \& Sons, Inc. $E$, Reprinted from Risold et al. (41), () 1997, with permission of Elsevier Science.

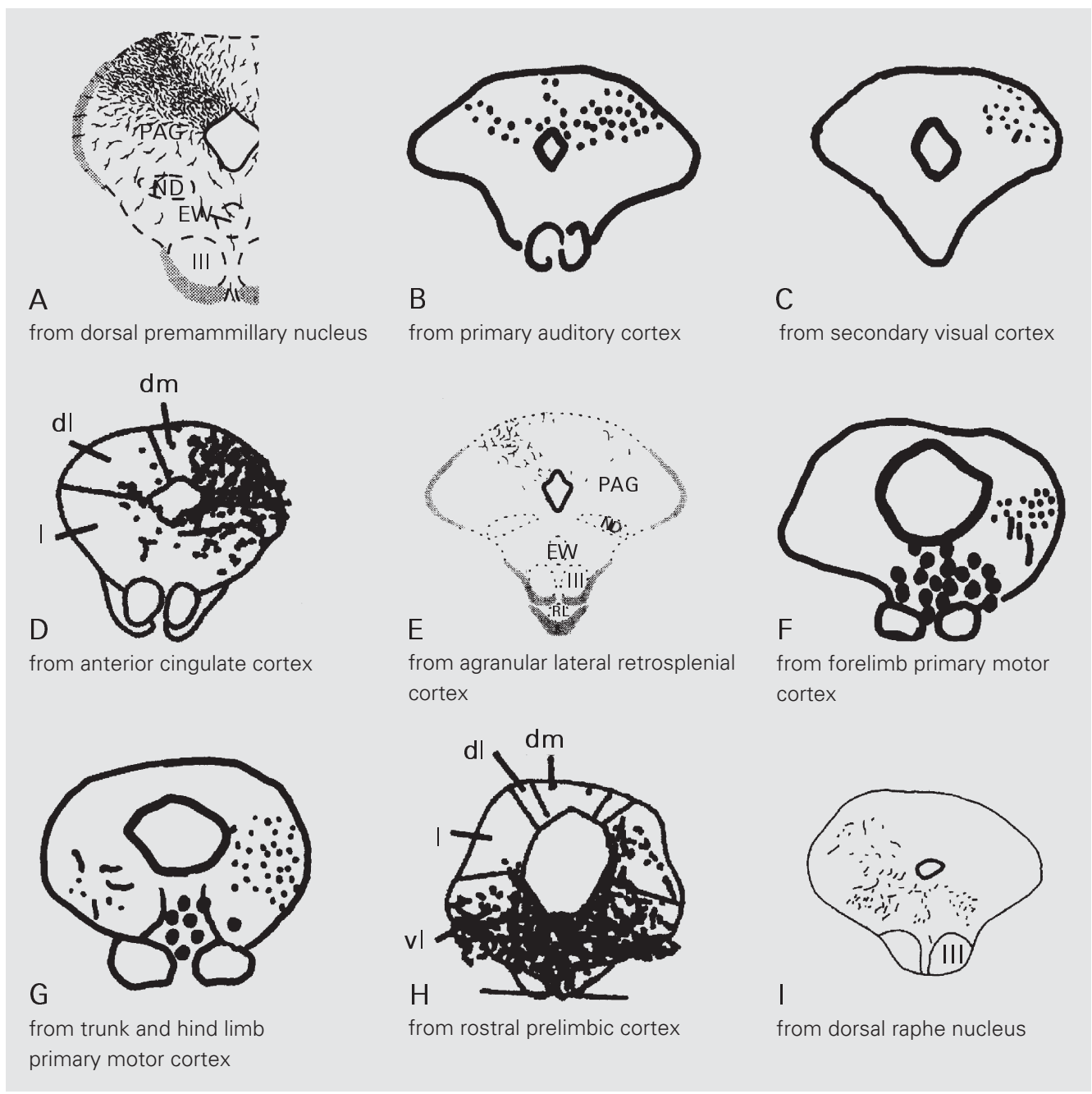


to hypothalamic nuclei related to defensive responses $(\mathrm{AHN}, \mathrm{DMH})$, while other regions have moderate (caudal dIPAG to AHN and DMH; vlPAG to DMH) or weak (caudal vlPAG to DMH) projections.

The AHN strongly projects to all PAG subdivisions (33). Another dense projection goes to the $\mathrm{VMH}$, which projects massively to the rostral dIPAG (29). The VMH also projects to various amygdalar nuclei, including the central nucleus (29). The AHN have reciprocal connections with the PMd, and also an indirect projection via the $\mathrm{VMH}$ (29). The PMd projects massively to the dlPAG (Figure 2A) and rostral PAG, and much less to other PAG subdivisions (34). In summary, the dlPAG is the PAG subdivision preferentially connected with the hypothalamic nuclei outlined above (Figure 3).

\section{Amygdala}

The amygdala and PAG only share connections through the central nucleus. These connections are direct and reciprocal, and both inputs and outputs preferentially involve the vlPAG (35) (Figure 4D). The inactivation of this path with lidocaine causes a drastic reduction of conditioned freezing (36). The only target structure of the central nucleus whose lesion has the same effect is the caudal PAG (5). The amygdala is related to conditioned and unconditioned fear (37), but not to unconditioned freezing behavior, since its excitotoxic lesion does not change freezing immediately after a foot shock (38), and even the conditioned fear deficit can be compensated for with overtraining (39). Thus, this structure might be better conceptualized as a perceptual interface than as an effector for the limbic system.

\section{Thalamus}

There is no consensus about where the projections from discrete PAG subdivisions terminate in the thalamus. Krout and Loewy

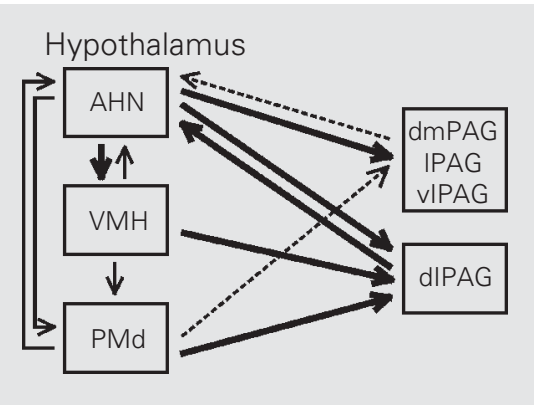

Figure 3. Main connections between hypothalamic behavioral defense nuclei and periaqueductal gray (PAG). The thickness of the arrows indicates the relative density of the projections. AHN, anterior hypothalamic nucleus; dIPAG, dmPAG, IPAG and vIPAG, dorsolateral, dorsomedial, lateral and ventrolateral PAG subdivisions, respectively; PMd, dorsal premammillary nucleus; $\mathrm{VMH}$, ventromedial hypothalamus.

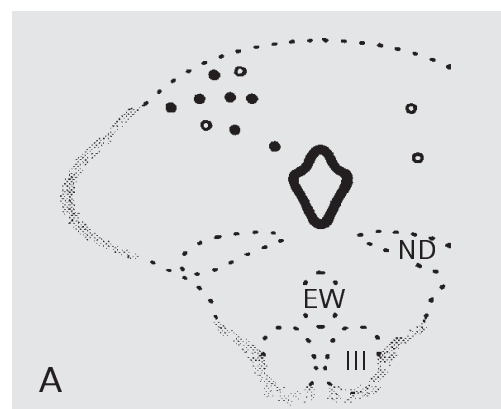

to nucleus reuniens

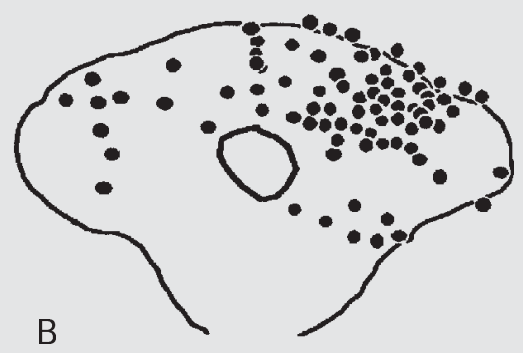

to nucleus cuneiformis
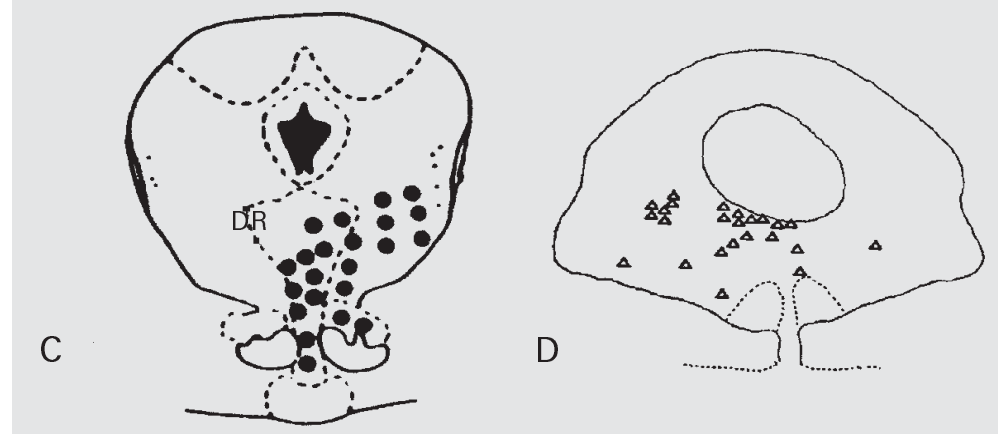

to median raphe nucleus

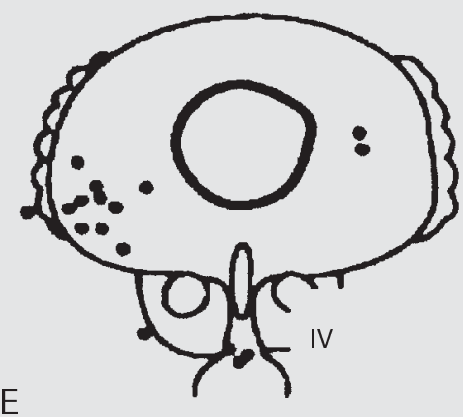

to spinal cord

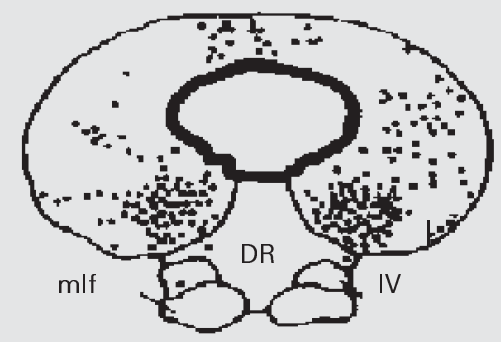

$\mathrm{F}$

to nucleus raphe pallidus

Figure 4. Periaqueductal gray neurons that project to other structures. III, oculomotor nucleus; IV, trochlear nucleus; DR, dorsal raphe nucleus; EW, Edinger-Westphal nucleus; mlf, medial longitudinal fasciculus; ND, nucleus of Darkschewitsch. A, Reprinted from Risold et al. (41) and F, from Hermann et al. (51), () 1997, with permission of Elsevier Science. B, Reprinted from Redgrave et al. (49), and E, from Mouton and Holstege (55), () 1988, 1994, with permission of Springer-Verlag. C, Reprinted from Marcinkiewicz et al. (44), and D, from Rizvi et al. (35), () 1989, 1991, with permission of Wiley-Liss, Inc., a subsidiary of John Wiley \& Sons, Inc. 
(40) observed that PAG terminations in the thalamus originate predominantly from the lateral PAG and vlPAG, innervating medial and intralaminar thalamic nuclei. In the same study, after microinjection of a retrograde tracer into the nucleus reuniens, all PAG subdivisions were stained, with clear predominance of the most caudal aspects of the lateral PAG. In contrast, in a similar study on the same nucleus, Risold et al. (41) observed clear-cut retrograde staining of the dIPAG (Figure 4A). The nucleus reuniens also receives strong projections from the AHN, VMH and PMd (41), the hypothalamic 'defense' nuclei proposed by Canteras (29).

\section{Cortex}

In the rat, primary motor areas of forelimbs (Figure 2F), hind limbs and trunk (Figure $2 \mathrm{G}$ ) project exclusively to the lateral PAG and vlPAG, while primary auditory (Figure 2B) and secondary visual (Figure 2C) cortices preferentially innervate the dIPAG (42). Perirhinal, anterior cingulate (43) (Figure 2D) and agranular lateral retrosplenial cortices (41) (Figure 2E) project almost exclusively to the dIPAG. Medial, ventrolateral, ventral, dorsolateral orbital, and dorsal and posterior insular agranular cortices project to the vlPAG (43). Prelimbic and infralimbic cortices are the borders of these topographic domains, with their rostroventral portions targeting the vlPAG (Figure $2 \mathrm{H}$ ) while dorsocaudally they project to the dIPAG. Thus, descending cortical projections are topographically distributed to distinct regions of the PAG.

Direct projections from PAG to cortex are not known. Nevertheless, escape behavior caused by dIPAG stimulation with nitric oxide donors leads to activation of the anterior cingulate (33), suggesting the existence of an ascending path functionally linking the two regions. The nucleus reuniens of the thalamus could be a relay station (41).

\section{Rostral raphe}

The median raphe nucleus has reciprocal direct connections with the vlPAG (44) (Figure 4C) and receives strong direct projections from the PMd. Recently, the median raphe nucleus has been related to context conditioning retention (45).

Dorsal raphe nucleus projections terminate densely in the vlPAG, but also in the dIPAG at the level of the oculomotor nucleus (46) (Figure 2I). Serotoninergic projections from dorsal raphe nucleus to dIPAG are inhibitory, and probably modulate the activity of neurons involved in defensive behavior (47). The vlPAG is the only PAG subdivision that sends direct projections to the dorsal raphe nucleus (48).

\section{Connections with lower structures}

The descending impulse flow to the motoneurons of the ventral horn of the spinal cord from the PAG travels through relay stations at the level of the cuneiform nucleus and caudal raphe.

Most of the PAG projections to the cuneiform nucleus arise from the dIPAG (49) (Figure 4B). Chemical stimulation of the cuneiform nucleus causes freezing and escape behavior, and its lesion blocks defense reactions produced by stimulation of the superior colliculus (50), a region that shares this projection with the dlPAG. Neurons in this nucleus are activated by exposure to predator odor (17) and stimulation of dorsal PAG (14) and medial hypothalamus (15).

Caudal raphe nuclei receive afferents preferentially from the dmPAG, lateral PAG and vlPAG, but not from the dIPAG (51). The caudal vlPAG contains the largest group of cells that send projections to the raphe nucleus (51) (Figure 4F). These nuclei also receive afferents from the cuneiform nucleus, but their precise topography has not yet been analyzed (52). Stimulation of neurons in this region causes immobility (53), and electrical 
stimulation provokes escape (54).

The vIPAG is the only PAG subdivision that sends direct projections to the ventral horn (55) (Figure 4E). Nevertheless, other subdivisions can reach somatic motoneurons through the nucleus raphe pallidus and obscurus (56).

\section{Synthesis}

We cannot say much about the dmPAG and lateral PAG on the basis of available information. These regions are clearly distinguishable by their cytoarchitecture and are separated by an NADPH-d-positive dIPAG, but at least in the context of the behavioral component of defensive responses in the rat there is no precise information on connections inherent to one of them. One possibility is the lateral PAG-thalamus projection, but this awaits confirmation. In contrast, one can readily distinguish the structures or regions that preferentially connect with the dlPAG and vlPAG. A summary of available data is presented in Table 1 .

The differential projection topography of the dIPAG and vlPAG may provide answers to some apparent inconsistencies reported in the literature. Below we deal with two issues that have arisen from this perspective.

\section{Dorsolateral and ventrolateral periaqueductal gray subdivisions and the control of the freezing response}

The vlPAG has been implicated in the mediation of conditioned freezing and quiescence. Its involvement in conditioned freezing is probably mediated by its exclusive connections with the central nucleus of the amygdala.

The dIPAG has also been related to the freezing behavior that occurs interspersed with flight. Rats, roaches and other animals alternate freezing and flight when under threat by predators, making their position in space less predictable and capture less likely. This freezing seems to be different from conditioned freezing because it outlasts vlPAG lesion (27) and is related to immediate danger, while the former is not. Both freezing and escape induced by immediate danger were virtually abolished after PMd lesion (29), a nucleus that preferentially projects to the dIPAG (32).

The amygdala has also been related to unconditioned fear, but its lesion does not affect the freezing response to immediate danger (38). Hence, it is wiser to propose a dissociation of dIPAG and vlPAG function as mediating the responses to immediate and cued danger, respectively, than one based on the conditioned/unconditioned dyad.

\section{The post-stimulation freezing}

After aversive dlPAG stimulation, rats freeze. This effect is resistant to context shift and does not last $24 \mathrm{~h}$, suggesting sensitization and not conditioning (25). The same is not observed with the vlPAG. Surprisingly, after escape provoked by vlPAG stimulation, the rat seems to be calm, as if no aversive event had taken place (26).

It is tempting to suggest an involvement of medial prefrontal and anterior cingulate

Table 1. Main connections between central nervous system structures and periaqueductal gray.

\begin{tabular}{llll}
\hline & Input to PAG & Two-way input & PAG output \\
\hline dIPAG & $\begin{array}{l}\text { Sensory cortex } \\
\text { Prefrontal cortex* } \\
\text { Cingulate cortex } \\
\text { Dorsal raphe }\end{array}$ & Hypothalamus** & $\begin{array}{l}\text { CnF } \\
\text { Thalamus*** }\end{array}$ \\
& Motor cortex & Amygdala & \\
vIPAG & Prefrontal cortex* & $\begin{array}{l}\text { Dorsal raphe } \\
\text { Insular cortex }\end{array}$ & $\begin{array}{l}\text { Caudal raphe } \\
\text { Ventral horn } \\
\end{array}$ \\
& Median raphe & Thalamus** \\
\hline
\end{tabular}

$\mathrm{CnF}$, cuneiform nucleus; PAG, periaqueductal gray; dIPAG, dorsolateral PAG; vIPAG, ventrolateral PAG.

* Distinct regions project preferentially to the dIPAG and VIPAG.

**For a more detailed summary of the connections between hypothalamus and PAG, see Figure 3.

***There is no consensus in the literature about the preferential PAG afferent domains. 
cortices in post-stimulation freezing through memory processes. These regions have a decisive role in short-term memory. Interference with this system prevents information from staying in memory after removal of associated environmental clues (57). Indeed, rats with infralimbic cortex lesion and submitted to an aversive conditioning procedure showed a great reduction in conditioned freezing and ultrasonic vocalizations (58). However, another study using a similar procedure in rats bearing lesions of the same region did not confirm this finding (59). The main difference between the two studies was that in the latter study freezing was measured during conditioned stimulus presentation, while in the former it was recorded immediately after the presentation was over. These regions are thus implicated in short-term memory. This also seems to be true regarding nociceptive stimulation. In fact, it has been shown that, after digital amputation, evoked responses to electrical stimulation of the contralateral digit in the anterior cingulate are enhanced. This effect lasts about 120 min, a temporal course again compatible with sensitization processes (60). Therefore, a participation of these cortical regions in the short-term storage of aversive information, as observed with the sustained freezing after the interruption of the dIPAG stimulation, needs to be considered.

\section{Conclusion}

In conclusion, the anatomo-functional organization of the PAG is consistent with the assumption that the PAG is a collection of separate functional entities with distinct ascending and descending connections and roles in different defensive responses. Besides being able to send projections to lower brainstem regions, both dIPAG and vlPAG are able to send information to structures located rostrally. Thus, the PAG is more than just a final common path for defensive responses. Finally, freezing induced by electrical stimulation of the dIPAG is not context sensitive, unlike that mediated by the vlPAG. Therefore, the defense reactions induced by activation of the dIPAG are independent of the neural mechanisms in the vlPAG involved in fear conditioning. The defensive behaviors generated at the dIPAG level are likely to represent unconditioned fear responses to impending danger, which have been implicated in panic disorder.

\section{References}

1. Hunsperger RW (1963). Comportements affectifs provoqués par la stimulation électrique du tronc cérébral et du cerveau antérieur. Journal de Physiologie, 55: 45-97.

2. Graeff FG (1994). Neuroanatomy and neurotransmitter regulation of defensive behaviors and related emotions in mammals. Brazilian Journal of Medical and Biological Research, 27: 811-829.

3. Tomaz C, Brandão M, Bagri A, Carrive P \& Schmitt P (1988). Flight behavior induced by microinjection of GABA antagonists into periventricular structures in detelencephalated rats. Pharmacology, Biochemistry and Behavior, 30: 337-342.

4. Blanchard DC, Williams G, Lee EMC \& Blanchard RJ (1981). Taming of wild Rattus norvegicus by lesions of the mesencephalic central gray. Physiological Psychology, 9: 157-163.

5. LeDoux JE, Iwata J, Cichetti P \& Reis DJ (1988). Different projections of the central amygdaloid nucleus mediate autonomic and behavioral correlates of conditioned fear. Journal of Neuroscience, 8: 2517-2529

6. Carrive P \& Bandler R (1991). Viscerotopic organization of neurons subserving hypotensive reactions within the midbrain periaqueductal gray: A correlative functional and anatomical study. Brain Research, 541: 206-215.

7. Bandler R \& Shipley MT (1994). Columnar organization in the midbrain periaqueductal gray: modules for emotional expression? Trends in Neurosciences, 17: 379-389.

8. Carrive $P$ (1993). The periaqueductal gray and defensive behaviour: functional representation and neuronal organization. Behavioural Brain Research, 58: 27-47.

9. Fanselow MS (1991). The midbrain periaqueductal gray as a coordinator of action in response to fear and anxiety. In: Depaulis A \& Bandler R (Editors), The Midbrain Periaqueductal Gray Matter. Plenum, New York.

10. Depaulis A, Keay KA \& Bandler R (1994). Quiescence and hyporeactivity evoked by activation of cell bodies in the ventrolateral periaqueductal gray of the rat. Experimental Brain Research, 99: 7583.

11. Carrive P (2000). Conditioned fear to environmental context: cardio- 
vascular and behavioral components in the rat. Brain Research, 858: 440-445.

12. Blanchard DC \& Blanchard RJ (1988). Ethoexperimental approaches to the biology of emotion. Annual Review of Psychology, 39: 43-68.

13. Morgan MM \& Carrive $P$ (2001). Activation of the ventrolateral periaqueductal gray reduces locomotion but not mean arterial pressure in awake, freely moving rats. Neuroscience, 102: 905-910.

14. Sandner G, Di Scala G, Rocha B \& Angst MJ (1992). C-fos immunoreactivity in the brain following unilateral electrical stimulation of the dorsal periaqueductal gray in freely moving rats. Brain Research, 573: 276-283.

15. Sandner G, Oberling P, Silveira MC, Di Scala G, Rocha B, Bagri A \& Depoortere $R$ (1993). What brain structures are active during emotion? Effects of brain stimulation elicited aversion on c-Fos immunoreactivity and behavior. Behavioural Brain Research, 58: 9-18.

16. Canteras NS \& Goto M (1999). Fos-like immunoreactivity in the periaqueductal gray of rats exposed to a natural predator. NeuroReport, 10: 413-418.

17. Dielenberg RA, Hunt GE \& McGregor IS (2001). "When a rat smells a cat": the distribution of Fos immunoreactivity in rat brain following exposure to a predatory odor. Neuroscience, 104: 1085-1097.

18. Carrive P, Leung P, Harris J \& Paxinos G (1997). Conditioned fear to context is associated with increased fos expression in the caudal ventrolateral region of the midbrain periaqueductal gray. Neuroscience, 78: 165-177.

19. Brandão ML, de Aguiar JC \& Graeff FG (1982). GABA mediation of the anti-aversive action of minor tranquilizers. Pharmacology, Biochemistry and Behavior, 16: 397-402.

20. Schenberg LC, Costa MB, Borges PCL \& Castro FS (1990). Logistic analysis of the defense reaction induced by electrical stimulation of the rat mesencephalic tectum. Neuroscience and Biobehavioral Reviews, 14: 473-479.

21. Krieger JE \& Graeff FG (1985). Defensive behavior and hypertension induced by glutamate in the midbrain central gray of the rat. Brazilian Journal of Medical and Biological Research, 18: 61-67.

22. Bandler R, Depaulis A \& Vergnes M (1985). Identification of midbrain neurones mediating defensive behaviour in the rat by microinjections of excitatory amino acids. Behavioural Brain Research, 15: 107119.

23. Schmitt P, Eclancher F \& Karli P (1974). Etude des systèmes de renforcement négatif et de renforcement positif au niveau de la substance grise centrale chez le rat. Physiology and Behavior, 12: 271-279.

24. Di Scala G, Mana MJ, Jacobs WJ \& Phillips AG (1987). Evidence of Pavlovian conditioned fear following electrical stimulation of the periaqueductal gray in the rat. Physiology and Behavior, 40: 55-63.

25. Vianna DML, Landeira-Fernandez J \& Brandão ML (2001). Dorsolateral and ventral regions of the periaqueductal gray matter are involved in distinct types of fear. Neuroscience and Biobehavioral Reviews, 25: 711-719.

26. Vianna DML, Graeff FG, Brandão ML \& Landeira-Fernandez J (2001). Defensive freezing evoked by electrical stimulation of the periaqueductal gray: comparison between dorsolateral and ventrolateral regions. NeuroReport, 12: 4109-4112.

27. Vianna DML, Graeff FG, Landeira-Fernandez J \& Brandão ML (2001). Lesion of the ventral periaqueductal gray reduces conditioned fear but does not change freezing induced by stimulation of the dorsal periaqueductal gray. Learning and Memory, 8: 164-169.

28. Schmitt $P$, Carrive $P$, Di Scala G, Jenck F, Brandão M, Bagri A, Moreau J-L \& Sandner G (1986). A neuropharmacological study of the periventricular neural substrate involved in flight. Behavioural
Brain Research, 22: 181-190.

29. Canteras NS (2002). The medial hypothalamic defensive system: hodological organization and functional implications. Pharmacology, Biochemistry and Behavior, 71: 481-491.

30. de Oliveira RW, Del Bel EA \& Guimarães FS (2000). Behavioral and c-fos expression changes induced by nitric oxide donors microinjected into the dorsal periaqueductal gray. Brain Research Bulletin, 51: 457-464.

31. Beck CHM \& Fibiger HC (1995). Conditioned fear-induced changes in behavior and in the expression of the immediate early gene c-fos: with and without diazepam pretreatment. Journal of Neuroscience, 15: 709-720.

32. Cameron AA, Khan IA, Westlund KN, Cliffer KD \& Willis WD (1995). The efferent projections of the periaqueductal gray in the rat: a Phaseolus vulgaris-leucoagglutinin study. I. Ascending projections. Journal of Comparative Neurology, 351: 568-584.

33. Semenenko FM \& Lumb BM (1992). Projections of anterior hypothalamic neurones to the dorsal and ventral periaqueductal grey in the rat. Brain Research, 582: 237-245.

34. Canteras NS \& Swanson LW (1992). The dorsal premammillary nucleus: an unusual component of the mammillary body. Proceedings of the National Academy of Sciences, USA, 89: 10089-10093.

35. Rizvi TA, Ennis M, Behbehani M \& Shipley MT (1991). Connections between the central nucleus of the amygdala and the midbrain periaqueductal gray: topography and reciprocity. Journal of Comparative Neurology, 303: 121-131.

36. Carrive P, Lee J \& Su A (2000). Lidocaine blockade of amygdala output in fear conditioned rats reduces fos-expression in the ventrolateral periaqueductal gray. Neuroscience, 95: 1071-1080.

37. Vazdarjanova A (2000). Does the basolateral amygdala store memories for emotional events? Trends in Neurosciences, 23: 345-346.

38. Antoniadis EA \& McDonald RJ (2001). Amygdala, hippocampus, and unconditioned fear. Experimental Brain Research, 138: 200-209.

39. Maren S (1999). Neurotoxic basolateral amygdala lesions impair learning and memory but not the performance of conditioned fear in rats. Journal of Neuroscience, 19: 8696-8703.

40. Krout KE \& Loewy AD (2000). Periaqueductal gray matter projections to midline and intralaminar thalamic nuclei of the rat. Journal of Comparative Neurology, 424: 111-141.

41. Risold PY, Thompson RH \& Swanson LW (1997). The structural organization of connections between hypothalamus and cerebral cortex. Brain Research. Brain Research Reviews, 24: 197-254.

42. Newman DB, Hileary SK \& Ginsberg CY (1989). Nuclear terminations of corticonuclear fiber systems in rats. Brain, Behavior and Evolution, 34: 223-264.

43. Floyd NS, Price JL, Ferry AT, Keay KA \& Bandler R (2000). Orbitomedial prefrontal cortical projections to distinct longitudinal columns of the periaqueductal gray in the rat. Journal of Comparative Neurology, 422: 556-578.

44. Marcinkiewicz M, Morcos R \& Chrétien M (1989). CNS connections with the median raphe nucleus: retrograde tracing with WGAapoHRP-gold complex in the rat. Journal of Comparative Neurology, 289: 11-35.

45. Avanzi $\vee$ \& Brandão ML (2001). Activation of somatodendritic 5$\mathrm{HT}_{1 \mathrm{~A}}$ autoreceptors in the median raphe nucleus disrupts the contextual conditioning in rats. Behavioural Brain Research, 126: 175184.

46. Vertes RP (1991). A PHA-L analysis of ascending projections of the dorsal raphe nucleus in the rat. Journal of Comparative Neurology, 313: 643-668.

47. Lovick TA (1994). Influence of the dorsal and median raphe nuclei on 
neurons in the periaqueductal gray matter: role of 5-hydroxytryptamine. Neuroscience, 59: 993-1000.

48. Kalén P, Karlson M \& Wiklund L (1985). Possible excitatory amino acid afferents to nucleus raphe dorsalis of the rat investigated with retrograde wheat germ agglutinin and $\mathrm{D}-\left[{ }^{3} \mathrm{H}\right]$ aspartate tracing. Brain Research, 360: 285-297.

49. Redgrave P, Dean P, Mitchell IJ, Odekunle A \& Clark A (1988). The projection from superior colliculus to cuneiform area in the rat. I. Anatomical studies. Experimental Brain Research, 72: 611-625.

50. Dean P, Redgrave P \& Mitchell IJ (1988). Organisation of efferent projections from superior colliculus to brainstem in rat: evidence for functional output channels. Progress in Brain Research, 75: 27-36.

51. Hermann DM, Luppi P-H, Peyron C, Hinckel P \& Jouvet M (1997). Afferent projections to the rat nuclei raphe magnus, raphe pallidus and reticularis gigantocellularis pars alpha demonstrated by iontophoretic application of choleratoxin (subunit b). Journal of Chemical Neuroanatomy, 13: 1-21.

52. Cowie RJ \& Holstege G (1992). Dorsal mesencephalic projections to pons, medulla, and spinal cord in the rat: limbic and non-limbic components. Journal of Comparative Neurology, 319: 536-559.

53. Morgan MM \& Whitney PK (2000). Immobility accompanies the antinociception mediated by the rostral ventromedial medulla of the rat. Brain Research, 872: 276-281.

54. Prado WA \& Roberts MHT (1985). An assessment of the antinociceptive and aversive effects of stimulating identified sites in the rat brain. Brain Research, 340: 219-228.

55. Mouton LJ \& Holstege G (1994). The periaqueductal gray in the cat projects to lamina VIII and the medial part of lamina VII throughout the length of the spinal cord. Experimental Brain Research, 101: 253-264.

56. Holstege JC \& Kuypers HGJM (1987). Brainstem projections to spinal motoneurons: an update. Neuroscience, 23: 809-821.

57. Goldman-Rakic PS (1992). Working memory and the mind. Scientific American, 267: 110-117.

58. Frysztak RJ \& Neafsey EJ (1991). The effect of medial frontal cortex lesions on respiration, "freezing", and ultrasonic vocalizations during conditioned emotional responses in rats. Cerebral Cortex, 1: 418-425.

59. Quirk GJ, Russo GK, Barron JL \& Lebron K (2000). The role of ventromedial prefrontal cortex in the recovery of extinguished fear. Journal of Neuroscience, 20: 6225-6231.

60. Wei F \& Zhuo M (2001). Potentiation of sensory responses in the anterior cingulate cortex following digit amputation in the anaesthetised rat. Journal of Physiology, 532: 823-833. 